\title{
Functionally Graded Panels: A Review
}

\author{
Sreeju Nair S B ${ }^{1}$ C. Pany ${ }^{2}$ \\ ${ }^{1}$ Department of Civil Engineering, PRS College of Engineering and Technology, Thiruvananthapuram, Kerala, India \\ ${ }^{2}$ Structural Entity,VSSC, Thiruvananthapuram, Kerala, India
}

To Cite this Article

Sreeju Nair S B, C. Pany, "Functionally Graded Panels: A Review", International Journal for Modern Trends in Science and Technology, 6(8): 36-43, 2020.

Article Info

Received on 02-June-2020, Revised on 26-June-2020, Accepted on 23-July-2020, Published on 28-July-2020.

\section{ABSTRACT}

Functionally gradedmaterials (FGMs) are not homogeneous materials. It consists of different(two or more) materials, engineered to have a continuously varying spatial composition profile. FGM is the one that can solve practical problems arising from the production and application of a new type of composite material. This paper describes the overview of FGM basic concepts, classification, properties, and its modeling which may focus on the static and dynamic analysis of functionally graded panels. The effective material properties of functionally graded materials for the panel are graded in the thickness direction from the bottom surface to the top surface according to the power-law distribution of volume fractions of the constituents. The use of structures like beams, plates, and shells, which are made from functionally graded (FG) materials, is increasing because of the smooth variation of material properties along with preferred directions. This variation gives continuous stress distribution in the FG structures. Therefore, an FGM can be effectively used in avoiding corrosion, fatigue, fracture, and stress corrosion cracking. The paper covers the literature study on static, buckling and free vibration, thermo-mechanical analysis of FGM panel. From this literature study it is found that, analysis of these problems is made using the constitutive relations and governing equations associated with the classical laminated theory structural model, the FSDT model, the HSDT model, Reissner and Sander theory, differential quadrature, finite element method and closed form solutions. Results are availableon different geometrical dimensional ratios variations, power-law index value $n$ variationsand simply supported,clamped, free edges boundary conditionswith its combinations for FG panels. Lesser literatures are available for different edge boundary conditions such as SCSC, CSCS,SSSC, SFSF, SSSF, SCSF on curved panelfor free vibration, buckling and thermo-structural analysis.

KEYWORDS: Functionally Graded Panels, Finite Element Modeling, End Constraints

\section{INTRODUCTION}

In material science functionally graded material may be characterized by the variation in structural composition gradually over volume, resulting in corresponding changes in the properties of the material. Within FGMs the different microstructural phases have different functions, and the overall FGMs attain the microstructural status from their property gradation. Composite materials allow distinct combinations giving hard, wear-resistant surface, and soft-core as per the functional requirement of the application. Heterogeneity, anisotropy, symmetry, and hierarchy are the main characteristics of composite materials reaping particular interest for various applications. High strength to stiffness ratio, greater resistance to fatigue, wear and corrosion, high reliability etc. are the advantages of composites over pure or alloyed metals. In spite of all these advantages, composite materials are subjected to the sharp transition of properties at the interface which can result in component failure 
(by delamination) at extreme working conditions. This drawback of conventional composites eliminated by a modified form of composites called FGMs. Material development from the past to the present and future is presented inFigure 1 .

These materials replace the sharp interface with gradient interface which results in a smooth transition of properties from one material to the other. FGMs are graded property materials and are used as medical implants, for thermal protection of space vehicles, as a thermoelectric converter for energy conservation, etc.

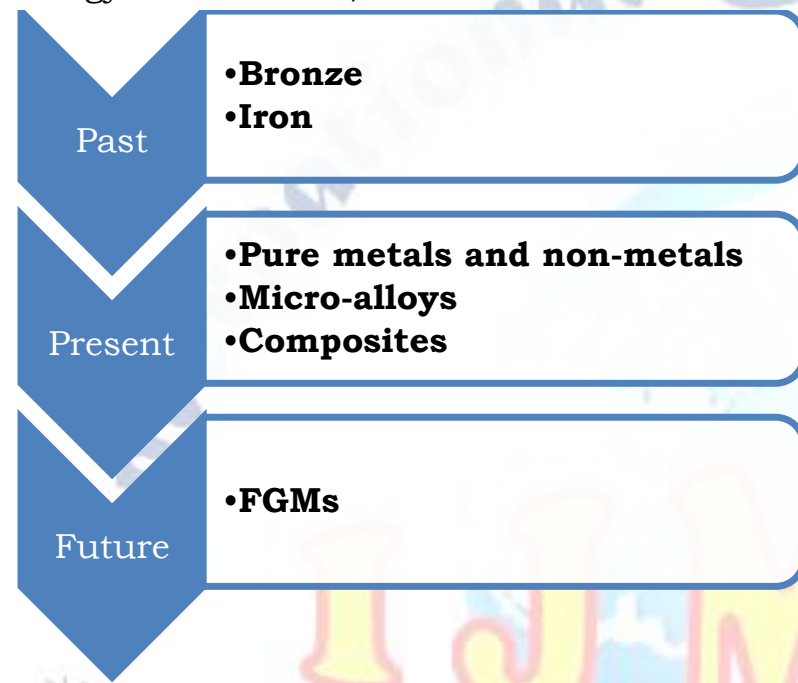

Figure 1. Flowchart showing the history of materials used in industry

FGM are the advanced composite materials made of two or more constituent phases which have a continuous and smooth variation of composition and material properties such as elasticity moduli, density and Poisson's ratio in preferred directions. These aspects provide a lot of advantages to FGM over conventional homogeneous materials in terms of optimization of material properties. This super composite materials shows continuous and discontinuous variation with regard to composition and microstructure as shown in Figure 2. The continuous change in the microstructure of FGM provides many advantages over fibre-reinforced laminated composite materials, such as a lesser probability of debonding at high thermalloading.
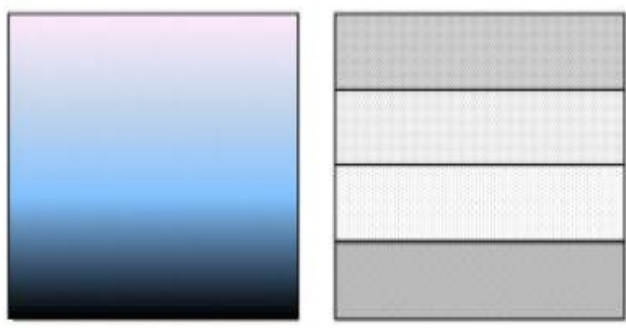

Figure 2. Functionally graded material property continuous and step-wise variation [1]
Nowadays the research has been carried out on FGMs intensively. FGMs are also a concern in the other fields such as industrial materials, optoelectronics, biomaterials, and energy materials. FGMs offer great promise in applications where the operating conditions are more severe. Potential applications include those structural and engineering uses that require combinations of hardness with toughness.Applications of FGMs have significant advantages in civil and mechanical systems including thermal systems (e.g. rocket heat shields, heat exchanger tubes, thermoelectric generators, wear-resistant linings, diesel and turbine engines, etc.). Various fields having advantages of using FGM are shown in figure 3.Fibre-reinforced polymer (FRP) is another application of FGMs for reinforcing concrete materials. The FRP materials improve the corrosion resistance of the steel and enhance the life cycle of the material strength.

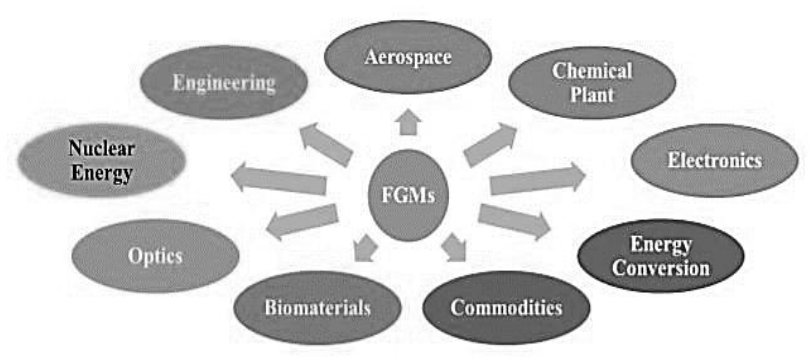

Figure 3. Applications of FGM

The finite element method (FEM) is a computational technique used to obtain approximate solutions of boundary value problems in engineering. Boundary value problems or field problems, is the domain of interest and most often represents a physical structure. The field variables are the dependent variables of interest governed by the differential equation. The boundary conditions are the specified values of the field variables (or related variables such as derivatives) on the boundaries of the field.

The progress in the theory of plate formulation from the year 1789-1988 is presented by Jemielitz[2]. These theories are grouped into layer-wise, zig-zag, and equivalent single-layer theories. The reviews of all equivalent single-layer theories for the analysis of laminated composite plates are presented recently by Sayyad et al [3]. Classical plate theory (CPT) is the simplest theory for the analysis of laminated composite plates but inaccurate for the thick plate due to neglect of the transverse shear deformation eff ect. 
The functionally graded (FG)plate considered in the present study(Figure 4) is a mixture of ceramic and metal. The top surface of the plate is made up of ceramic and the bottom surface is made up of metal. The following mixture law is used to obtain properties of the material through the thickness of FG plates.

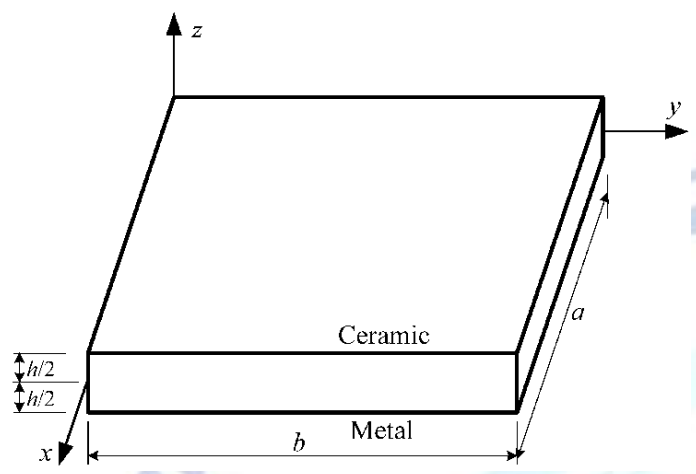

Figure 4. Rectangular plate where $\mathrm{a} / \mathrm{b}>1$

Properties of each layer having thickness $z$ ofan FG plate can be determined by considering the volume fraction eqs. (1) to (5) corresponding to different gradient indices.

$$
\begin{aligned}
\mathrm{E}_{(Z)} & =\left(\mathrm{E}_{\mathrm{C}}-\mathrm{E}_{\mathrm{M}}\right) \mathrm{V}_{\mathrm{f}(\mathrm{Z})}+\mathrm{E}_{\mathrm{M}} \\
\mathrm{p}_{(\mathrm{Z})} & =\left(\mathrm{p}_{\mathrm{C}}-\mathrm{p}_{\mathrm{M}}\right) \mathrm{V}_{\mathrm{f}(\mathrm{Z})}+\mathrm{p}_{\mathrm{M}} \\
\mu_{(\mathrm{Z})}= & \left(\mu_{\mathrm{C}}-\mu_{\mathrm{M}}\right) \mathrm{V}_{\mathrm{f}(\mathrm{Z})}+\mu_{\mathrm{M}} \\
k_{(z)} & =\left(k_{c}\right)\left[1+\frac{3\left(k_{M}-k_{C}\right)\left(1-V_{f(Z)}\right)}{3 k_{c}\left(1-V_{f(Z)}\right)+\left(k_{M}+2 k_{c}\right) V_{f(Z)}}\right] \\
& \alpha_{(Z)} \\
& =\left(\alpha_{C}-\alpha_{M}\right) \\
& +\left(\alpha_{M}\right)\left[\frac{\left(1-V_{f(Z)}\right) V_{f(Z)}\left(\alpha_{C}-\alpha_{M}\right)\left(k_{M}-k_{C}\right)}{\left(k_{M}-k_{C}\right) V_{f(Z)}+k_{M}+\left[\frac{3 k_{M} k_{C}}{4 G_{M}}\right]}\right]
\end{aligned}
$$

where $E_{m}, p_{m}, \mu_{m}, k_{m}, \alpha_{m}$ and $E_{c}, p_{c}, \mu_{c}, k_{c}, \alpha_{c}$ are the Young's moduli, density, poisson's ratio, thermal conductivity and coefficient of thermal expansion of metal and ceramic, respectively.

The mechanical properties of anFGM plate change continuously from one surface to another through its thickness direction according to the power lawis expressed as

$\mathrm{V}_{\mathrm{f}(\mathrm{Z})}=\left[\frac{Z}{h}+\frac{1}{2}\right]^{n}$
$\mathrm{V}_{\mathrm{f}(z)}$ is the volume fraction and $\mathrm{n}$ is the volume fraction exponent which takes values equal to zero or greater than zero. The value of $n$ equals to zero represents a fully ceramic plate.

\section{LITERATURE SURVEY}

Nath[4] used an efficient finite-difference technique (based on displacement potential formulation) to solve a simply supported composite beam under uniformly distributed loading. The beam is analyzed considering plane stress condition and orthotropic properties. Vosoughiet al. [5] presents a finite element (FE), genetic algorithm (GA) and particle swarm optimization (PSO) methods are mixed to maximize buckling load of the stiffened laminated composite plate via finding optimum fibers orientation of the plate. For employing the method, the finite element method is utilized to obtain buckling load of the plate and the $\mathrm{FE}$ solution is coupled with the improved version of the genetic algorithm. Carrera and Demasi[6,7] deal with the formulation of finite plate elements for an accurate description of stress and strain fields in multilayered, thick plates subjected to static loadings in the linear, elastic cases. A zig-zag form and interlaminar continuity are addressed in the considered formulations. Variational methods such as the principle of virtual displacements (PVD), and the Reissner mixed variational theorem (RMVT) are employed to derive finite element matrices. They also present numerical evaluations related to the multilayered plate elements. Two-dimensional modelings with linear and higher-order (up to fourth order) expansion in the $z$-plate layer thickness direction has been implemented for both displacements and transverse stresses. Layer-wise as well as equivalent single-layer modelings are considered on both frameworks of the principle of virtual displacements and Reissner mixed variational theorem. Dewanganet al. [8] investigated the geometrical linear and nonlinear flexural behavior of laminated composite plates under uniformly distributed load. The modeling of the plate is done using ANSYS 15.0 based on the First-order Shear Deformation Theory (FSDT) and von-Karman nonlinear strain. An eight nodes element with six degrees of freedom at each node has been considered for discretization purposes. The convergence and comparison test of the present model has been done by considering results available in published literature. Isoldiet al. [9] analyzed the linear static and dynamic behavior of 
thin laminate composite structures using the FEM. Triangular elements (three nodes) with six degrees of freedom per node (three translation and three rotation components) are used. Karet al. [10] demonstrated and discussed the deformation characteristics of FGM flat and curved (cylindrical and spherical) panels for different volume fractions, thickness ratios, curvature ratios, and boundary conditions. To achieve this, the finite element solutions are obtained using ANSYS parametric design language (APDL) code using an eight-noded serendipity element (SHELL281) with 48 degrees of freedom. Bending responses of FGM flat and curved (cylindrical and spherical) panels are examined. Hassan and Keles[11] worked on the modeling of FGM using ANSYS APDL. ANSYS does not provide a direct way to model variation in material properties as a function of position. ANSYS offers the option of temperature-dependent properties; and it also offers the ability to assign temperature values according to the position; joining these two abilities offered by ANSYS. So, the material properties would be assigned based on position. Martinez-Pañeda[12]investigated the numerical implementation of functionally graded properties in the context of the FEM. The elastic properties (macroscopic) variation inherent to FGMs is introduced at the element level by means of the two most commonly used schemes: (i) nodal based gradation, and (ii) Gauss integration point-based gradation. These formulations are extensively compared by solving a number of paradigmatic boundary value problems for which analytical solutions can be obtained.

Abrate[13] studied free vibrations, buckling, and static deflections of functionally graded plates in which material properties vary through the thickness. It is shown that all other parameters keeping the same, the natural frequencies of FG plates are always proportional to those of homogeneous isotropic plates and that the proportionality constant can be easily be predicted. Similar results are obtained for buckling and static deflection cases. Therefore, one can predict the behavior of FG plates knowing that of similar homogeneous plates. Ferreiraet al. [14] analyzed static deformations of FG plate performed by using the collocation method(meshless method), the radial basis functions, and a higher-order shear deformation theory. The approach is allowed a fast and simple domain and boundary discretization. They choose the shape parameter in the radial basis functions by an optimization procedure based on the cross-validation technique and use the Mori-Tanaka homogenization technique to deduce the effective properties of FG materials. Ramu and Mohanty[15] have carried out a modal analysis of an FGM plate to determine its natural frequencies and mode shapes by using FEM \& Kirchhoff plate theory. The natural frequencies and mode shapes of rectangular FGM plate are obtained for various edge boundary conditions. Natural frequencies for CCCC boundary conditions are found highest. When the power-law index value $\mathrm{n}$ increases, the natural frequencies all the different edge boundary conditions reduce.Shegokar and Budruk[16] presented the stochastic bending response of FGMs shell panel under mechanical and thermal loadings. The formulation is presented based on higher-order shear deformation theory (HSDT) with von-Karman nonlinear strain kinematics. The assumptions considered is material properties are independent and graded in the thickness direction based on a simple power-law distribution in terms of the volume fraction of the constituents. Shao et al. [17] carried out a three-dimensional thermomechanical analysis of functionally graded cylindrical panels subjected to nonuniform mechanical and transient thermal loads. Mechanical and thermal properties are assumed as temperature-independent and varying continuously in the radial direction. Employing Laplace transform and the series solving method of an ordinary differential equation(s), solutions of time-dependent temperature and thermomechanical stress in FGM cylindrical panels are obtained. Momennia and Akbarzadeh[18] investigated the mechanical behavior of rectangular and circular plates made of FGMs. The analysis is based on the finite element approach using Abaqus, and those analytical solutions reported in the literature. Furthermore, some finite element results for cylindrical and conical shells are given in this investigation. Sharma et al. [19] worked to perform a thermo-mechanical analysis of the FGM square laminated plate made of Aluminum / Zirconia and compare it with pure metal and ceramic. The assumption made on plates are, to be isotropic, two constituents material distribution across the thickness, and Young's modulus of elasticity of the plate to vary by a power-law distribution following the volume fractions of the constituents. Alikaramiand Parvizi[20] presented an exact analytical elastoplastic solution for a thick-walled cylinder made of FGMs subjected to combined 
pressure and thermal loading. It is assumed that the cylinder is bonded at both ends, the material is radially graded and having elastic perfectly plastic behavior. A comparison of the present theoretical results with those from a finite element simulation illustrates good agreements observed between them. Gurol[21] proposed a new beam element that is based on force formulation for modeling elastic and inelastic analysis of beams with FGMs. The attempt of producing FGM arose from mixing two materials in such a way that both of them preserve their physical, mechanical, and thermal properties most effectively. Khan et al. [22] presented a 1D Finite Element model for static response and free vibration analysis of the FGM beam. The FE model is based on efficient zig-zag theory (ZIGT) with two noded beam element having four degrees of freedom per node. Linear interpolation is used for the axial displacement and cubic Hermite interpolation is used for the deflection. Uysal[23] presented the mechanical deformation behavior of shear deformable functionally graded ceramic-metal plates. Theoretical formulations are based on the power-law theory when building up FGM. The mechanical properties of the plate are graded in the thickness direction according to a power-law. Displacement and stress are obtained using the FEM.

Thai and Choi[24] presented analytical solutions for bending, buckling, and vibration analyses of thick rectangular plates with various boundary conditions using two variables refined plate theory. The theory accounts for the parabolic variation of transverse shear stress through the thickness of the plate without using the shear correction factor. In addition, it includes only two unknowns and similar to the classical plate theory in many aspects such as equations of motion, boundary conditions, and stress resultant expressions. The deflection, buckling load, and natural frequency closed-form solutions are presented for rectangular plates with two opposite sides simply supported and the other two sides having arbitrary boundary conditions. Srinivast[25] presents a unified exact analysis for the statics and dynamics of a class of thick laminates. A 3D linear small deformation theory of elasticity solution is developed for the bending, vibration, and buckling of thick orthotropic rectangular laminated plates with simply supported boundary conditions. Thick orthotropic plates are very stiff compared to thin plates which shows frequency responses in descending trend. Fukunaga and Sekine[26] present an optimization approach for symmetrically laminated plates to maximize buckling loads under combined loading. The coupling between bending and twisting is taken into consideration in the buckling analysis. Using lamination parameters, the effect of bending-twisting coupling on the buckling is discussed for different boundary conditions(simply supported or clamped edges). The bending-twisting coupling reduces the buckling loads as compared with those of especially orthotropic laminate without coupling. Wang[27] proposed a new set of grid points for applying differential quadrature (DQ) to the analysis of structural problems. The accuracy and convergence of differential quadrature for buckling analysis of laminated plates are discussed for a variety of buckling problems (composite laminated plates with various boundaries, constraints under uniaxial, biaxial, and combined uniaxial and shear loadings). From the results, the accuracy of differential quadrature with proposed grid spacings is satisfactory. Sahu and Datta[28] analyzed the dynamic stability behavior of laminated composite curved panels with cutouts subjected to in-plane static and periodic compressive loads using the FEM. A generalized Sanders' shear deformable theory with tracers is used in this study. Numerical results obtained for vibration and buckling of composite panels with cut-outs compare well with literature. Based on this study, the excitation frequency decreases with the introduction of cutouts. Instability occurs for further mass reduction. Ravikiran and Ganesan[29] presented the free vibration and linear thermal buckling analysis for FG cylindrical shells with clamped-clamped boundary condition based on temperature-dependent material properties. The material properties of FGM shells are assumed to vary smoothly and continuously across the thickness. The thermal buckling temperature mainly depends on the coefficient of thermal expansion. Huu et al. [30] developed an analytical investigation on free vibration of a simply supported functionally graded (FG) doubly curved shell panels resting on elastic foundation in the thermal environment. Heat conduction and temperature-dependent material properties are used. The temperature field is assumed to be a uniform distribution over the shell surface and varied in the thickness direction only. Numerical results validated that material volume fraction index, geometrical parameters, and temperature 
change have an adverse effect on the natural frequencies of the given panel. Vishesh and Subrata[31] investigated free vibration responses of shear deformable FG single/doubly curved panels under a uniform, linear, and nonlinear temperature fields. The micromechanical material model of FGM is computed using the Voigt model in conjunction with the power-law distribution to achieve continuous gradation. The material properties are assumed to be the function of temperatures. frequency responses are very much dependent on thickness ratio $(\mathrm{a} / \mathrm{h})$, aspect ratio $(\mathrm{a} / \mathrm{b})$, power-law indices, and temperature load irrespective of panel geometries. Kar and Panda[32] investigated the buckling behavior of functionally graded cylindrical panels under thermal loading. In functionally graded material, material properties vary smoothly from the metal phase to the ceramic phase. In this study, the effective material properties of the functionally graded panels are considered as temperature-dependent and the gradation is taken in the transverse direction according to the power-law distribution of volume fractions of each constituent. The critical buckling load increases with increase in power-law indices and aspect ratios and decrease with increase in thickness ratios and curvature ratios (R/a). Manish and Kamlesh[33] considered power law (P-FGM), sigmoid law (S-FGM), and exponential distribution (E-FGM) for volume fraction distribution of FG plates subjected to transverse UDL and point load. The bending response is found to be intermediate to those of metal and ceramic plates. Bending response for S-FGM remains closer for various values of $\mathrm{n}$ as compared to that of $\mathrm{P}-\mathrm{FGM}$. The bending response of E-FGM is similar to the behavior of P-FGM. Hasan[34] investigated the shape effect on the free vibration behavior of FG plates. Circular, elliptical, annular, rectangular, square, skew, and equilateral triangular plates with the same surface area and thickness are compared for simply supported and clamped boundary conditions. Lowest six vibrations modes were taken and for both boundary conditions, the annular plate has the highest (5 to7 times) frequency values than other FG plates. Circular plates have the lowest values. Also, the frequency values show increasing from simply supported to clamp. Shahidzadeh and fattahi [35] conducted a modal analysis of the FGM plate containing the ceramics phase of the $\mathrm{Al} 2 \mathrm{O} 3$ and metal phase of stainless tell 304 using ABAQUS. Young modulus and density are varying in the thickness direction.
$\mathrm{FE}$ analysis was carried out in a fully clamped condition for different values of $\mathrm{n}$. There is a decrease in natural frequency with an increase of $n$ since the amount of ceramic phase decreases while the amount of metal phase increases leading to decrease in stiffness. Farhad et al. [36] investigated the buckling behavior of plates subjected to shear and edge compression. The effects of thickness, slenderness ratio, plate aspect ratio, boundary condition, and loading are numerically studied. The buckling coefficient in the plate with boundary condition (SSSS) is more than the decrease in the buckling coefficient in the plate with boundary condition (CCCC). Hosseini et al. [37] presented analytical solutions for free vibration analysis of moderately thick rectangular plates resting on Winkler and Pasternak elastic foundations having two opposite edges simply supported and all possible combination of free, simply supported and clamped boundary conditions other two edges for various aspect ratios, gradient indices, and thickness to length ratios. Manish et al. [38] worked on parametric studies by varying volume fraction distribution and boundary conditions of FG plates. Only mechanical deformation of FG ceramic-metal square plate, under various boundary conditions (SSSS, CCCC, SCSC, CFCF, CCFF, CCSS, SSFF, SSSC, SSSF, SSCF), has analyzed.

Talha and Singh[39]are studied the static and free vibration analysis of functionally graded material (FGM) plates using HSDT with modified transverse displacement in a FEM. When the volume fraction index ' $n$ ' increases, the non-dimensional central deflection to given plate thickness ratio increases. Similarly, as the aspect ratio, 'a/b' (ratio of smaller side to bigger) increases, the non-dimensional central deflection decreases. The frequency parameter increases with the increase in flat plates aspect ratio ' $a / b$ ' and ' $a / h$ ' (ratio of smaller side to thickness). Similarly with an increase in volume fraction index ' $n$ ' the frequency parameter decreases. The frequency parameter for all edges simply-supported (SSSS) is lower than all edges clamped (CCCC). Lidiya Kurpa et al.[40] applied the $\mathrm{R}$-functions theory and Ritz approach for analysis of free vibrations of laminated FG shallow shells with different types of curvatures and complex planforms. Effects of power-law exponents, thickness schemes, and different boundary conditions (clamped, simply supported, free edge, and their combinations) are studied for shells with the clamped cutout of the complex shape. 
Tahouneh and M.H.Naei[41] have carried out free vibration analysis of thick laminated curved panels with finite length, based on the 3D elasticity theory. The proposed approach is a generalized 2D differential quadrature method. It makes possible vibration analysis of cylindrical panels with two opposite axial edges simply supported and arbitrary boundary conditions including clamped, simply supported, and free at the curved edges. The material properties vary continuously through the thickness of the layer according to a 3 parameter power-low distribution. It is assumed that the inner surfaces of the FG sheets are metal-rich and outer surfaces of the layers can be metal-rich, ceramic rich, or made of a mixture of two constituents. The effects of geometrical, material parameters and the boundary conditions on the frequency parameters of the laminated FG panels are studied. The results show that the outer FGM Layers have significant effects on the vibration behavior of cylindrical panels. It has been concluded the non-dimensional natural frequency of laminated panels with ceramic layers rich is higher than the non-dimensional frequency of homogeneous layers of metal. Further, the non-dimensional frequency decreases rapidly with the increase of the length-to-mean radius ratio $(\mathrm{L} / \mathrm{R})$ and then remains almost constant for the long cylindrical panels. This study serves as a benchmark for validating numerical methods or 2D theories analysis of laminated curved panels.

\section{CONCLUSION AND FUTURE WORKS}

This literature study covers the worked carried out on FGM panels (flat and curved panels) on static, free vibration, buckling, and thermal analysis.Numerical illustrations and results with respect to dimensional ratios and gradient indices are presented.

Further, mostly literatures are available on SSSS, CCCC, free edges boundary conditions and its combinationfor FG panels. And literature data on the effect of different edge boundary conditions for the FG curved panels are not many for free vibration, buckling, thermo-structural analysis.

The future work is aimed at the analysis (static, free vibration, buckling, thermo-structural) of FG flat/curved thin/thick panels considering different end constraints (SSSS, CCCC, SCSC, SSSC, SFSF, SSSF, SCSF) for various functionally graded combinations with varying essential geometrical parameters (i.e. aspect ratio, radius to length ratio, varying thickness along length or breadth) and volume fraction index. These results will be useful for designers, field engineer working in this area to understand the static, free vibration, buckling, and thermo-structural characteristics

\section{REFERENCES}

[1] Williams S. Ebhota, T. J. (2016). "Casting and Application of functionally graded metal matrix composites."IntechOpen, 13,

59-86,dx.doi.org/10.5772/intechopen.71225

[2] Jemielitz, G. (1993). "On the Winding Paths of the Theory of Plates." Journal of Theoretical and Applied Mechanics.

[3] A. S. Sayyad, Y. M. G. (2014). "Buckling and free vibration analysis of orthotropic plates by using exponential shear deformation theory." Latin American Journal of Solids and Structures, 11, 1298-1314.

[4] Nath, S. K. D. (2014). "A Finite Difference Solution of a Simply Supported Beam of Orthotropic Composite Materials Using Displacement Potential Formulation." Chinese journal of Engineering ,2014, http://dx.doi.org/10.1155/2014/961503

[5] Vosoughi, A. R., Darabi, A., Anjabin, N., and Topal, U. (2017). "A mixed finite element and improved genetic algorithm method for maximizing buckling load of stiffened laminated composite plates." AerospaceScience and Technology, Elsevier Masson SAS, 70,378-387, http://dx.doi.org/10.1016/j.ast.2017.08.022

[6] Carrera, E., and Demasi, L. (2002a). "Classical and advanced multilayered plate elements based upon PVD and RMVT . Part 1 : Derivation of ÿnite element matrices." 231(April 2001),Int. J. Numer. Meth. Engng 2002, 55,191-231, DOI: $10.1002 /$ nme.492

[7] Carrera, E., and Demasi, L. (2002b). "Classical and advanced multilayered plate elements based upon PVD and RMVT . Part 2: Numerical implementations." 291(April 2001), Int. J. Numer. Meth. Engng 2002, 55, 253-291, DOI: $10.1002 /$ nme.493

[8] Dewangan, S., Mahapatra, T. R., and Sharma, N. (2016). "Flexural Analysis of Laminated Composite Flat Panel."Journal of Material Science and Mechanical Engineering (JMSME), 3(3), 219-223.

[9] Isoldi, L. A., Awruch, A. M., Morsh, I. B., Roberto, P., and Teixeira, D. F. (2010). "Linear static and dynamic analysis of thin laminated composite structures with a triangular finite element" Vetor, Rio Grande, 20, 2, 38-57.

[10] Kar, V. R., Panda, S. K., Tripathy, P., Jayakrishnan, K., Rajesh, M., Karakoti, A., and Manikandan, M. (2018). Deformation characteristics of functionally graded composite panels using finite element approximation. Modelling of Damage Processes in Biocomposites, Fibre-Reinforced Composites and Hybrid Composites, Elsevier,https://doi.org/10.1016/B978-0-08-102289-4.0 0012-6

[11] Hassan, A., and Keles, I. (2017). "FGM Modelling using dummy thermal loads, applied with ansys APDL." Journal of selcuk international science and technology, 1(1), 10-16.

[12] Martinez-Pañeda, E. (2019). "On the finite element implementation of functionally graded materials." Materials 2019, 28, doi: $10.3390 / \mathrm{ma} 12020287$

[13] Abrate, S. (2006). "Free vibration, buckling, and static deflections of functionally graded plates." Composites 
Science and Technology,elsevier Inc.,66,2383-2394, doi:10.1016/j.compscitech.2006.02.032

[14] Ferreira, A. J. M., Roque, C. M. C., Jorge, R. M. N., Fasshauer, G. E., Batra, R. C., and Batra, R. C. (2007). "Analysis of functionally graded plates by a robust meshless method." (December 2014), Mechanics of Advanced Materials and Structures, 14, 577-587, DOI: 10.1080/15376490701672732

[15] Ramu, I., and Mohanty, S. C. (2014). "Modal analysis of Functionally Graded material Plates using Finite Element Method." Procedia materials science,6,460-467,doi: 10.1016/j.mspro.2014.07.059.

[16] Shegokar, N. L., and Budruk, C. (2014). "Thermomechanical nonlinear analysis of functionally graded thermomechanical nonlinear analysis of functionally graded cylindrical shell panel with random system parameter." (June 2012), Mechanics of Nano, Micro and Macro Composite Structures

[17] Shao, Z. S., Wang, T. J., and Ang, K. K. (2007). "Transient thermomechanical stresses of functionally graded cylindrical panels." (October 2007), AIAA JOURNAL,45(10), DOI: 10.2514/1.24328.

[18] Momennia, S., and Akbarzadeh, A. H. (2011). "Analysis of functionally graded rectangulare and circular plates using finite element method." 16th International Conference on Composites Structures, 1, 1-2.

[19] Sharma, M., Bhandari, M., and Purohit, K. (2013). "Deflection of Functionally Gradient Material Plate.", Global Journal of Researches in Engineering Mechanical \& Mechanics, 13(7), 3-9.

[20] Alikarami, S., and Parvizi, A. (2017). "Elasto-plastic analysis and finite element simulation of thick-walled functionally graded cylinder subjected to combined pressure and thermal loading." Science and Engineering of Composite Materials, 24(4), 609-620, DOI 10.1515/secm-2015-0010

[21] Gurol, T. (2014). "Finite element modeling of beams with functionally graded materials." (February 2014), School of Natural And Applied Sciences Of Middle East Technical University, 86.

[22] Khan, A. A., Alam, M. N., UR Rahman, N., and Wajid, M. (2016). "Finite element modelling for static and free vibration response of functionally graded beam." Latin American Journal of Solids and Structures, 13(4), 690-714, http://dx.doi.org/10.1590/1679-78252159

[23] Uysal, M. U. (2013). "Investigation of thermal and mechanical loading on functional graded material plates." International Journal of Aerospace and Mechanical Engineering, 7(11), 1219-1225.

[24] Thai, H., and Choi, D. (2013). "Analytical solutions of refined plate theory for bending, buckling and vibration analyses of thick plates." Applied mathematical modelling, elsevier Inc., 37(18-19), 8310-8323, http://dx.doi.org/10.1016/j.apm.2013.03.038.

[25] Srinivast, S. (1970). "Bending, Vibration And Buckling Of Simply Supported Thick Orthotropic Rectangular Plates And Laminates"Int. J. Solids Structures, 6, 1463-1481.

[26] Fukunaga, H., and Sekine, H. (1995). "Buckling design of symmetrically laminated plates using lamination parameters." Computers \& Structures, 57(4), 643-649

[27] Wang, X. (1995). "Differential quadrature for buckling analysis oflaminated plates." Computers \& Structures, 57(4), 715-719

[28] Sahu, S. K., and Datta, P. K. (2004). "Dynamic Stability of Laminated Composite Curved Panels with Cutouts." Journal of Engineering Mechanics, ASCE,
129(11),1245-1253

10.1061/(ASCE)0733-9399(2003)129:11(1245).

[29] Ã, R. K., and Ganesan, N. (2006). "Buckling and free vibration analysis of functionally graded cylindrical shells subjected to a temperature-specified boundary condition." Journal of Sound and Vibration, 289, 450-480, doi:10.1016/j.jsv.2005.02.034.

[30] Huu, Q., Huan, T., Duong, T., and Minh, T. (2018). "Free vibration analysis of functionally graded doubly curved shell panels resting on elastic foundation in thermal environment." International journal of advanced structural engineering, Springer Berlin Heidelberg, 10(3), 275-283, https://doi.org/10.1007/s40091-018-0197-x.

[31] Vishesh Ranjan Kara Subrata Kumar Panda. (2015). "Free vibration responses of temperature dependent functionally graded curved panels under thermal environment." Latin American Journal of Solids and Structures, 12, 2006-2024, http://dx.doi.org/10.1590/1679-78251691

[32] Kar, V. R., and Panda, S. K. (2014). "Thermal buckling of temperature dependentfunctionally graded cylindrical panel." 5th International \& 26th All India Manufacturing Technology, Design and Research Conference (AIMTDR 2014), 183-(1-5).

[33] Bhandari, M., and Purohit, K. (2014). "Static Response of Functionally Graded Material Plate under Transverse Load for Varying Aspect Ratio." International Journal of Metals, 2014, 1-11, http://dx.doi.org/10.1155/2014/980563.

[34] Kurtaran, H. (2017). "Shape Effect on Free Vibration of Functionally Graded Plates." International Journal of Engineering \& Applied Sciences, 6(4), 52-52.

[35] S.J. Shahidzadeh Tabatabaei, A. M. Fa. (2016). "FEM for Modal Analysis of FGM." World Acadamy of science, engineering and technology, 10(2), 339-343.

[36] Riahi, F. (2017). "Buckling Stability Assessment of Plates with Various Boundary Conditions Under Normal and Shear Stresses." Engineering, Technology \& Applied Science Research, 7(5), 2056-2061.

[37] Hosseini-Hashemi, S., Rokni Damavandi Taher, H., Akhavan, H., and Omidi, M. (2010). "Free vibration of functionally graded rectangular plates using first-order shear deformation plate theory." Applied Mathematical Modelling, 34(5), 1276-1291, doi:10.1016/j.apm.2009.08.008.

[38] Bhandari, M. (2014). "Comparison of Functionally Graded Material Plate with Metaland Ceramic Plateunder Transverse Loadfor Various Boundary Conditions."International Journal of Computer Applications (0975 - 8887), National Conference on Advances in Technology \& Applied Sciences (NCATAS2014), 25-29.

[39] Mohammad Talha, B.N. Singh.(2010)“ Static response and free vibration analysis of FGMplates using higher order shear deformation theory.", Applied mathematical modeling,34,3991-4011.doi:10.1016/j.apm.2010.03.034.

[40] Lidiya, Kurpa,Tetyana Shmatko,Jan Awrejcewicz(2019). "Vibration analysis of laminated functionally graded shallow shellswith clamped cutout of the complex form by the Ritz method and the R-functions theory". Latin American Journal of Solids and Structures, 16(1), e95,dx.doi.org/10.1590/1679-78254911.

[41] V. Tahouneh, M.H.Naei. (2016) Semi-analytical solution for free vibration analysis of thick laminated curved panels with power-law distribution fg layers and finite length via two- dimensional gdq method. Journal of solid mechanics, 8(2), 334-347. 